Larry Joel Goldstein

Nagoya Math. J.

Vol. 53 (1974), 235-237

\title{
ERRATA FOR DEDEKIND SUMS FOR A FUCHSIAN GROUP, I
}

\section{LARRY JOEL GOLDSTEIN}

Dr. Bruce Berndt has pointed out to me several errors in the calculation of the Dedekind sums $S_{N}(\sigma)$ for the principal congruence subgroups. Several errors are of the nuisance variety and have no effect whatever on the calculation. However, there is one error, which can be corrected, but which leads to a somewhat different formula for $S_{N}(\sigma)$.

First the minor errata:

p. 33, l. $-12 \quad \sigma_{i} \sigma_{i}^{-1} \rightarrow \boldsymbol{R}$ should read $\sigma_{i}^{-1} \Gamma \sigma_{i} \rightarrow \boldsymbol{R} / \boldsymbol{Z}$.

p. 33, l. -11 $S_{r, i}(\sigma)$ should $\operatorname{read} S_{\Gamma, i}(\sigma)(\bmod Z)$.

p. 34, l. 3 Omit sentence beginning 'However'.

p. 34, l. $8 \quad$ Should $\operatorname{read} S_{\Gamma, i}(\sigma \tau) \equiv S_{\Gamma, i}(\sigma)+S_{r, i}(\tau)(\bmod Z)$.

p. 43, l. $-101-Q^{r}$ (resp. $1-\bar{Q}^{r}$ ) should read $1-\zeta_{N} Q^{r}$ (resp. $\left.1-\zeta_{N}^{-1} Q^{r}\right)$. $1 / 4 \pi$ should read $1 / 4 \pi \alpha_{N}$.

p. $43, l .-7$ The correct value for $\sigma(z)$ is $(a / c)+i u$. This value must be inserted in equations (46), (47), (49) and (53). However, there is no change in any of the computations.

p. 44, l. 9 The correct value for $Q_{0}$ is $e^{-2 \pi i N d / c}$.

p. 44, l. -7 The correct value for $Q_{1}$ is $e^{-2 \pi u N}$.

The serious error is that equation (54) is incorrect owing to an error in the preceding derivation. However, the infinite series of (54) may be easily summed as follows:

$$
\begin{aligned}
& \sum_{r=1}^{\infty} \frac{\zeta_{N}^{j} W^{j r}-\zeta_{N}^{-j} W^{-j r}}{r} \\
&=2 i \sum_{r=1}^{\infty} \frac{\sin \{(2 \pi j / N)+(2 \pi N a j r / c)\}}{r} \\
&=2 i\left[\sin \left(\frac{2 \pi j}{N}\right) \sum_{r=1}^{\infty} \frac{\cos (2 \pi N a j r / c)}{r}\right.
\end{aligned}
$$




$$
\begin{gathered}
\left.+\cos \left(\frac{2 \pi j}{N}\right) \sum_{r=1}^{\infty} \frac{\sin (2 \pi N a j r / c)}{r}\right] \\
=-2 i \sin \left(\frac{2 \pi j}{N}\right) \log \left|2 \sin \left(\frac{\pi N a j}{c}\right)\right|-2 \pi i \cos \left(\frac{2 \pi j}{N}\right)\left(\left(\frac{N a j}{c}-\frac{1}{2}\right)\right)
\end{gathered}
$$

However, if $\eta$ is a $k^{\text {th }}$ root of $1, \eta \neq 1$ we clearly have

$$
\frac{1}{1-\eta}=\sum_{j=0}^{k-1} \eta_{j}
$$

Therefore, since $\zeta_{N} W^{r}$ is a $|c| N^{\text {th }}$ root of 1 , we see that if $\zeta_{N} W^{r} \neq 1$, then

$$
\lim _{u \rightarrow 0}\left\{\frac{1}{1-\zeta_{N} W^{r} Q_{1}^{r}}-\frac{1}{1-\zeta_{N}^{-1} W^{-r} Q_{1}^{r}}\right\}=\sum_{j=0}^{|c| N-1}\left\{\zeta_{N}^{j} W^{j r}-\zeta_{N}^{-j} W^{-j r}\right\}
$$

Thus,

$$
\begin{aligned}
\lim _{u \rightarrow 0} \operatorname{Im} \log \eta_{N}\left(\frac{a}{c}+i u\right)= & \frac{a}{4 \pi \alpha_{N} c}-\frac{1}{2 i} \sum_{j=0}^{|c| N-1} \sum_{r=1}^{\infty} \frac{\zeta_{N}^{j} W^{j r}-\zeta_{N}^{j} W^{-j r}}{r} \\
= & \frac{a}{4 \pi \alpha_{N} c}+\sum_{j=0}^{|c| N-1} \sin \left(\frac{2 \pi j}{N}\right) \log \left|2 \sin \left(\frac{\pi N a j}{c}\right)\right| \\
& +\pi \sum_{j=0}^{|c| N-1} \cos \left(\frac{2 \pi j}{N}\right)\left(\left(\frac{N a j}{c}-\frac{1}{2}\right)\right) .
\end{aligned}
$$

However,

$$
\begin{aligned}
\sum_{j=0}^{|c| N-1} \sin \left(\frac{2 \pi j}{N}\right) & \log \left|2 \sin \left(\frac{\pi N a j}{c}\right)\right| \\
& =\sum_{j=0}^{|c| N-1} \sin \left(\frac{2 \pi(|c| N-j)}{N}\right) \log \left|2 \sin \left(\frac{\pi N a(|c| N-j)}{c}\right)\right| \\
& =-\sum_{j=0}^{|c| N-1} \sin \left(\frac{2 \pi j}{N}\right) \log \left|2 \sin \left(\frac{\pi N a j}{c}\right)\right|
\end{aligned}
$$

so that

$$
\sum_{j=0}^{|c| N-1} \sin \left(\frac{2 \pi j}{N}\right) \log \left|2 \sin \left(\frac{\pi N a j}{c}\right)\right|=0
$$

Therefore, we have,

$$
\lim _{u \rightarrow 0} \operatorname{Im} \log \eta_{N}\left(\frac{a}{c}+i u\right)=\frac{a}{4 \pi \alpha_{N} c}+\pi \sum_{j=0}^{|c| N-1} \cos \left(\frac{2 \pi j}{N}\right)\left(\left(\frac{N a j}{c}-\frac{1}{2}\right)\right) .
$$

Thus, finally, we have 
THEOREM 5-4: Let $\sigma=\left(\begin{array}{ll}a & b \\ c & d\end{array}\right) \in \overline{\Gamma(N)}$. Then the Dedekind sum $S_{N}(\sigma)$ is given by

$$
\begin{aligned}
S_{N}(\sigma) & =\frac{b}{4 \pi^{2} \alpha_{N}} \text { if } c=0 \\
& =\frac{1}{4 \pi^{2} \alpha_{N}}\left(\frac{a+d}{c}\right)-\frac{1}{4} \frac{c}{|c|}+\sum_{j=0}^{|c| N-1} \cos \left(\frac{2 \pi j}{N}\right)\left(\left(\frac{N a j}{c}-\frac{1}{2}\right)\right) \quad(c \neq 0),
\end{aligned}
$$

where $\alpha_{N}=\left(3 / \pi^{2}\right) \sigma(N)\left\{N^{2} \prod_{P \mid N}\left(1-p^{-2}\right)\right\}^{-1}$. In particular, $S_{N}(\sigma)$ is an algebraic number belonging to the $N^{\text {th }}$ cyclotomic field.

The author is indebted to Dr. Berndt for pointing out the error in the original paper.

University of Maryland 